\title{
Properties of electromagnetic fields generated by tsunami first arrivals: Classification based on the ocean depth
}

\section{$\operatorname{AUTHOR}(S)$ :}

Minami, Takuto; Toh, Hiroaki; Tyler, Robert H.

\section{CITATION:}

Minami, Takuto ...[et al]. Properties of electromagnetic fields generated by tsunami first arrivals: Classification based on the ocean depth. Geophysical Research Letters 2015, 42(7): 2171-2178

ISSUE DATE:

2015-04-16

URL:

http://hdl.handle.net/2433/229490

\section{RIGHT:}

(C)2015. American Geophysical Union.; The full-text file will be made open to the public on 27 October 2015 in accordance with publisher's 'Terms and Conditions for Self-Archiving'. 


\section{Geophysical Research Letters}

\section{RESEARCH LETTER}

10.1002/2015GL063055

Key Points:

- Tsunami-generated magnetic fields are strongly controlled by the ocean depth

- Magnetic tsunami signals become largest around an ocean depth of $1.5 \mathrm{~km}$

- Magnetic fields observable prior to tsunami arrivals change with the ocean depth

Supporting Information:

- Text S1

Correspondence to:

T. Minami,

minami@kugi.kyoto-u.ac.jp

Citation:

Minami, T., H. Toh, and R. H. Tyler (2015), Properties of electromagnetic fields generated by tsunami first arrivals: Classification based on the ocean depth, Geophys. Res. Lett., 42, 2171-2178, doi:10.1002/2015GL063055.

Received 12 JAN 2015 Accepted 5 MAR 2015 Accepted article online 8 MAR 2015 Published online 1 APR 2015

\section{Properties of electromagnetic fields generated by tsunami first arrivals: Classification based on the ocean depth}

\author{
Takuto Minami ${ }^{1,2}$, Hiroaki Toh ${ }^{3}$, and Robert H. Tyler ${ }^{2}$ \\ ${ }^{1}$ Graduate School of Science, Kyoto University, Kyoto, Japan, ${ }^{2}$ NASA Goddard Space Flight Center, Greenbelt, Maryland, \\ USA, ${ }^{3}$ Data Analysis Center for Geomagnetism and Space Magnetism, Kyoto University, Kyoto, Japan
}

\begin{abstract}
Tsunami flow coupled with the geomagnetic field generates electric currents and associated magnetic fields. Although electromagnetic (EM) tsunami signals can be used for analysis and even forecasting tsunami propagation, the dynamically self-consistent effect of shoaling water depth on the fluid + electrodynamics has not been adequately clarified. In this study, we classify tsunami EM phenomena into three cases based on the ocean depth and find that the deeper ocean results in stronger self-induction due to the increase in both tsunami phase velocity and ocean conductance. In this deep-ocean case, the phase lead of the vertical magnetic variation relative to the sea surface elevation is smaller, while an initial rise in the horizontal magnetic component becomes observable prior to tsunami arrival. Furthermore, we confirm that the enhancement of tsunami height in shallower oceans shifts the ocean depth supplying maximum amplitudes of tsunami magnetic fields from approximately $2.0 \mathrm{~km}$ to $1.5 \mathrm{~km}$.
\end{abstract}

\section{Introduction}

Electrically conductive seawater moving in the geomagnetic main field generates electric currents and secondary magnetic fields. Since Toh et al. [2011] first reported tsunami signals identified in seafloor electromagnetic (EM) observations, an increasing number of EM data associated with tsunamis have been reported. Most of these were obtained at the seafloor at the time of the recent extreme tsunamis, i.e., the 2010 Chile and the 2011 Tohoku earthquake tsunamis [e.g., Sugioka et al., 2014; Zhang et al., 2014a]. Tsunami-generated EM (TGEM) signals can be used not only to analyze tsunami events but also to predict real-time tsunami propagations. Of particular importance for hazard mitigation are the TGEM fields associated with the leading part of the tsunami wave, hereafter referred to as the "first arrivals." Although previous analytical solutions in the frequency domain are applicable to TGEM phenomena [e.g., Larsen, 1971; Tyler, 2005; Ichihara et al., 2013], we do not have a general insight into EM variations at the seafloor associated with first arrivals of destructive tsunamis, because (1) the first arrivals are inherently transient and difficult to study in the frequency domain and (2) the effect of the ocean depth on TGEM fields has never been discussed in detail. For specific TGEM events, three-dimensional (3-D) time-domain simulations may address these issues by following the recent developments in TGEM simulation techniques [e.g., Zhang et al., 2014b]. However, it will also be possible to understand basic characteristics of TGEM fields associated with tsunami first arrivals by combining analytical solutions in the frequency domain and two-dimensional (2-D) numerical experiments in the time-domain [Minami and Toh, 2013].

In this paper, we provide a new insight into the TGEM fields associated with tsunami first arrivals in terms of the ocean depth that strongly controls the TGEM phenomena. In the following, we first classify TGEM fields into three fundamental cases with the aid of analytical solutions of TGEM fields. Second, we investigate EM fields associated with tsunami first arrivals, using 2-D time-domain numerical simulations with solitary waves propagating over a flat seafloor. Finally, we examine how TGEM fields are caused by tsunamis when propagating through different ocean depths.

\section{Theory: Classification Based On the Ocean Depth}

Many researchers have derived theoretical expressions applicable to TGEM fields. For instance, Larsen [1971] derived a detailed relationship between intermediate/long waves and EM fields assuming a realistic 1-D conductivity structure beneath the seafloor, while Tyler [2005] derived a simpler expression applying the long-wave dispersion relationship and assuming half-space insulator on both sides of the ocean layer. 
Recently, Ichihara et al. [2013] derived an analytical solution assuming long waves and a homogeneous half-space conductor beneath the seafloor. In order to introduce a new way to classify TGEM fields, here we derive a new analytical solution of TGEM fields, assuming a linear dispersive tsunami. The governing equation of TGEM fields adopted here is the induction equation in terms of the tsunami-generated vertical magnetic component $\left(b_{z}\right)$ for only vertically variable conductivity $(\sigma=\sigma(z))$ :

$$
\frac{\partial b_{z}}{\partial t}=-\nabla \cdot\left(F_{z} \mathbf{u}_{H}\right)+K \nabla^{2} b_{z}
$$

Here $\mathbf{u}=\left(\mathbf{u}_{H}, u_{z}\right)$ is the oceanic velocity vector and $\mathbf{F}=\left(\mathbf{F}_{H}, F_{z}\right)$ is the vector of the geomagnetic main field, where the subscript $H$ and $z$ denote the horizontal vector and the vertical component, respectively. $K=(\mu \sigma)^{-1}$ is the magnetic diffusivity, where $\mu$ is the magnetic permeability. In equation (1), we have already decomposed the magnetic field $\mathbf{B}=\mathbf{F}+\mathbf{b}$ into the steady main field part $\mathbf{F}$ and the tsunami-generated part $\mathbf{b}$ and assumed that $|\mathbf{b}| \ll|\mathbf{F}|, \nabla^{2} \mathbf{F}=0, \nabla \times \mathbf{F}=0$, and $\left|F_{Z} \mathbf{u}_{H}\right| \gg\left|\mathbf{F}_{H} u_{z}\right|$. If we adopt a linear dispersive wave (LDW) over a constant ocean depth ( $z=0$ and $z=-h$ correspond to the sea surface and the seafloor, respectively), a homogeneous conductivity inside the ocean layer $(\sigma)$, and a homogeneous half-space conductor beneath the seafloor $\left(\sigma_{e}\right)$, we can solve equation (1) by assuming both $b_{z}$ and $F_{Z} \mathbf{u}_{H} \propto e^{i(\boldsymbol{k} \cdot \boldsymbol{x}-\omega t)}$, where $\mathbf{x}$ is the horizontal position vector, while $\omega$ and $\mathbf{k}$ are the angular frequency and the horizontal wave number vector of the tsunami in concern, respectively. It follows a relationship between $b_{z}$ and the sea surface elevation $(\eta)$ as

$$
\frac{b_{z}}{F_{z}}=C_{\mathrm{LDW}}\left(\omega, h, \sigma, \sigma_{e}\right) \times \frac{k \eta}{\sinh (k h)} \cdot(\mathrm{z}=-h) .
$$

Here $C_{\text {LDW }}$ is a complex transfer function (see supporting information for details of $C_{\mathrm{LDW}}$ ). If we further assume that (1) the tsunami can be approximated by linear nondispersive (long) waves, namely, $k h \ll 1,(2)$ the skin depth of seawater, $\delta(=\sqrt{2 K / \omega})$, is much larger than the ocean depth, $h$, and (3) $\sigma_{e}=0 \mathrm{~S} / \mathrm{m}$, we can reduce equation (2) to the simpler formula by Tyler [2005]

$$
\frac{b_{z}}{F_{z}}=\left(\frac{c}{c+i c_{d}}\right) \times \frac{\eta}{h} \cdot(\mathrm{z}=-h) .
$$

In the coefficient converting $\eta / h$ to $b_{z} / F_{z \prime} c(=\sqrt{g h})$ is the phase velocity of the long-wave tsunami, and $c_{d}(=2 K / h)$ is the lateral magnetic diffusion velocity, where $g$ is the gravitational acceleration. It has been found that equation (3) is still applicable to real TGEM signals observed at deep oceans [e.g., Ichihara et al., 2013]. From equations (2) and (3), we can calculate the phase lead of $b_{z} / F_{z}$ against $\eta$,

$$
\theta=-\arg \left(b_{z} / F_{z} \cdot 1 / \eta\right) \text {. }
$$

For equation (3), $\theta$ is reduced to $\tan ^{-1}\left(c_{d} / c\right)$. When $\theta=90^{\circ}$, the $b_{z} / F_{z}$ variation precedes $\eta$ by one quarter of a period since we assumed $b_{z}, \eta \propto e^{-i \omega t}$ and $F_{z}$ to be a real number (steady), while $b_{z} / F_{z}$ is in phase with $\eta$ when $\theta=0^{\circ}$.

Now we further investigate the dependence of TGEM fields on the ocean depth by utilizing equations (2)-(4). For the most general applicability, the dependence in forms of nondimensional parameters is sought. Using equations (2) and (3), we calculated the dependence of $\theta$ and $\left|b_{z} / F_{z} \cdot L / \eta\right|$ on the dimensionless ocean depth, $h / L$, as shown in Figure 1. Here we introduced a new length scale, $L \equiv(2 K / \sqrt{g})^{2 / 3}$, such that $c / c_{d}=(h / L)^{3 / 2}$. Assuming $(g, \mu, \sigma)=\left(9.8 \mathrm{~m} / \mathrm{s}^{2}, 4 \pi \times 10^{-7} \mathrm{H} / \mathrm{m}, 4.0 \mathrm{~S} / \mathrm{m}\right)$, we obtain $L \approx 2.53 \mathrm{~km}$. Figure 1 clearly shows that TGEM phenomena strongly depend on the ocean depth. The dependence can be understood by effects of the magnetic diffusion and the self-induction. In the magnetic induction equation, $\partial \mathbf{B} / \partial \mathbf{t}=\nabla \times(\mathbf{v} \times \mathbf{B})-\nabla \times(K \nabla \times \mathbf{B})$, the scaling amplitude of the self-induction term, $\partial \mathbf{B} / \partial t$, is determined by that of the diffusion term, $\nabla \times(K \nabla \times \mathbf{B})$, when normalized by the amplitude of the source term, $\nabla \times(\mathbf{v} \times \mathbf{B})$. For a homogeneous conductivity in the ocean, the scaling amplitude of the diffusion term is given by

$$
-\nabla \times(K \nabla \times \mathbf{B})=K \nabla^{2} \mathbf{B} \sim K\left(\frac{1}{h^{2}}+\frac{1}{\lambda^{2}}\right)|\mathbf{B}| \sim \frac{K}{h^{2}}|\mathbf{B}|,
$$

since the tsunami wavelength $(\lambda \sim 100 \mathrm{~km})$ is usually much larger than the ocean depth $(h<10 \mathrm{~km})$. Equation (5) means that as $h$ increases, the influence of the diffusion term attenuates, while the self-induction term, $\partial \mathbf{B} / \partial t$, must become larger to balance the source term. 




(a)

Dimensionless ocean depth, $h / L$

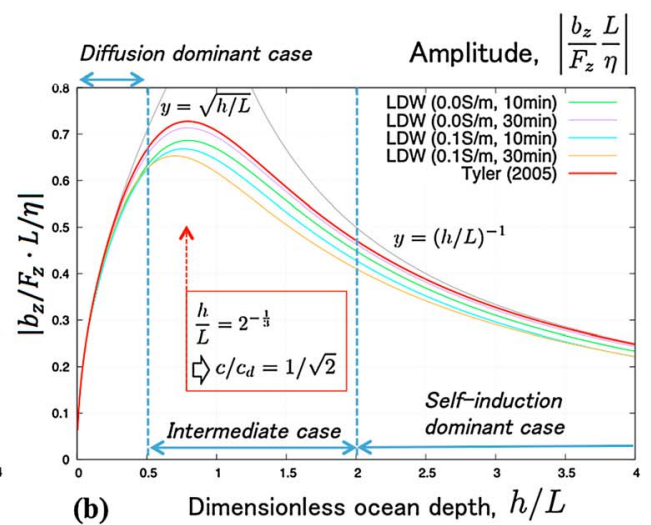

Figure 1. The phase lead of $b_{z} / F_{z}$ relative to (a) the sea surface elevation $(\eta), \theta$, and (b) the dimensionless amplitude $\left|b_{z} / F_{z} \cdot L / \eta\right|$, as functions of the dimensionless ocean depth, $h / L$. The Self-induction dominant and Diffusion dominant cases were defined for the depths which provide $\theta \leq 20^{\circ}$ and $70^{\circ} \leq \theta$, respectively. The red line was calculated using equation (3), while the others with keys of "LDW $\left(\sigma_{e}, T\right)$ " were calculated using equation (2). $\sigma_{e}$ and $T$ are the homogeneous half-space conductivity beneath the seafloor and the tsunami period, respectively. In Figure $1 \mathrm{~b}$, the red line has a peak where $h / L=2^{-\frac{1}{3}} \approx 0.7937$, viz., $c / c_{d}=1 / \sqrt{2}$, and asymptotes $y=\sqrt{h / L}$ and $y=(h / L)^{-1}$ in the Diffusion dominant case and Self-induction dominant case, respectively. In the LDW profiles of Figures $1 \mathrm{a}$ and $1 \mathrm{~b}$, we adopted $(g, \mu, \sigma)=\left(9.8 \mathrm{~m} / \mathrm{s}^{2}, 4 \pi \times 10^{-7} \mathrm{H} / \mathrm{m}, 4.0 \mathrm{~S} / \mathrm{m}\right)$, thereby $L$ was fixed approximately to $2.53 \mathrm{~km}$.

Here we define three fundamental cases for TGEM fields, "Diffusion dominant case," "Intermediate case," and "Self-induction dominant case," assigning 0 to 20 and 70 to $90^{\circ}$ phase leads to "Self-induction" and "Diffusion dominant case," respectively. Under this definition, dimensionless ocean depths $(h / L)$ smaller than approximately 0.5 corresponds to the Diffusion dominant case, and those larger than approximately 2.0 to the Self-induction dominant case (see Figure 1a). Using this classification, we can characterize response curves in Figure $1 \mathrm{~b}$ by functions which have peaks in the Intermediate case, and asymptote $y=(h / L)^{1 / 2}$ and $y=(h / L)^{-1}$ in the Diffusion dominant case and Self-induction dominant case, respectively.

In order to understand these characteristics in Figure 1b, we now rewrite equation (3) as

$$
\begin{aligned}
& b_{z}=\frac{\mu}{2} \cdot \frac{1}{Q+i} \cdot \int_{-h}^{0} i_{Q=0} \mathrm{~d} z \quad(z=-h), \\
& \text { where }\left\{\begin{array}{l}
Q=c / c_{d} \propto h^{\frac{3}{2}} \\
\int_{-h}^{0} i_{Q=0} \mathrm{~d} z \propto h^{\frac{1}{2}} .
\end{array}\right.
\end{aligned}
$$

Here $i_{Q=0}\left(=\sigma F_{z} c \eta / h\right)$ is the electric current density related to the source electromotive force, and $Q\left(=c / c_{d}\right)$ is hereafter referred to as the self-induction parameter whose magnitude relative to unity is a measure of the importance of the self-induction. Note that $Q$ in equation (6) is equivalent to the $Q$ value for a resistor-inductor circuit, $Q \equiv i\left(i_{Q=0}-i_{\text {net }}\right) / i_{\text {net }}$, where $i_{\text {net }}$ is the net electric current density, which is easily proved by using the expressions for TGEM fields, $i_{Q=0}=\sigma F_{z} c \eta / h$ and $i_{\text {net }}=i \sigma c_{d} b_{z}$. Equation (6) indicates that $b_{z}$ is controlled by the two competing terms: the conversion efficiency related to the self-induction, $1 /(Q+i)$, and the vertically integrated source current density, $\int_{-h}^{0} i_{Q=0} \mathrm{~d} z$, where the magnitude of the former decreases monotonically with the ocean depth, while that of the latter increases. Now we can interpret the features found in Figure $1 \mathrm{~b}$ using equation (6). In the Diffusion dominant case $(h / L<0.5)$, insignificant self-induction, $Q \ll 1$, leads to $1 /(Q+i) \sim-i$ such that $\theta \sim 90^{\circ}$ and $\left|b_{z} L / F_{z} \eta\right| \propto h^{1 / 2}$, which corresponds to the increase of $\left|\int_{-h}^{0} i_{Q=0} \mathrm{~d} z\right|$ with the ocean depth. In the Intermediate case $(0.5<h / L<2)$; however, the effect of the self-induction becomes comparable to that of the diffusion, viz., $Q \sim 1$. In this case, the decrease rate of $|1 /(Q+i)|$ exceeds the increase rate of $\left|\int_{-h}^{0} i_{Q=0} \mathrm{~d} z\right|$ as the ocean depth increases, which results in the peaks of $\left|b_{z} L / F_{z} \eta\right|$ around $h / L=2^{-\frac{1}{3}} \sim 0.7937$, viz., $Q=1 / \sqrt{2}$, which means $h \approx 2.0 \mathrm{~km}$ when $L=2.53 \mathrm{~km}$. In the Self-induction dominant case $(2<h / L)$, the self-induction becomes much more important than the diffusion, viz., $1 \ll Q$, which leads to $1 /(Q+i) \sim Q^{-1} \propto h^{-3 / 2}$ so that $\theta \sim 0^{\circ}$ and $\left|b_{z} L / F_{z} \eta\right| \propto h^{-3 / 2} \cdot h^{1 / 2}=h^{-1}$. 
Since the features in Figure $1 \mathrm{~b}$ was explained by equation (6), here we consider how the ocean depth physically affects the self-induction effect from the perspective of $Q=c / c_{d}$. First, we consider the effect of $c=\sqrt{g h}$. Originally, $c$ appeared as the phase velocity of the source oceanic flow, $\omega / k$, in the derivation of equation (3). Under the long-wave approximation, $c$ was reduced to the function of the ocean depth only, $\sqrt{g h}$. The phase velocity of tsunamis become faster with increasing ocean depth, which causes the stronger self-induction effect due to more rapid changes of the magnetic fields. Hence, the contribution of $c$ stems from the hydrodynamic property of tsunamis alone. Second, we investigate the contribution of $c_{d}$. Although there could be several possible interpretations, one of the reasonable explanations for $c_{d}$ is that $c_{d}=2 K / h=2 / \mu /(\sigma h)$ reflects the effect of the conductance of the ocean, $\tau \equiv \sigma h$. As $\tau$ increases with the ocean depth, the magnetic diffusion is more weakened relative to the self-induction current. We can regard $c_{d}$ as the efficiency of the EM diffusion. Hence, we can conclude that the magnitude of the self-induction, i.e., $Q=c / c_{d}$, increases with the ocean depth, due to the growth of the tsunami phase velocity (increase in $c$ ) and that of the conductance of the ocean layer (decrease in $c_{d}$ ). In the following section, we explain how tsunamis generate EM fields in the three cases described in this section.

\section{Numerical Experiments Using 2-D Solitary Waves}

We conducted 2-D simulations using solitary plane waves in order to investigate how EM fields are generated by tsunami first arrivals in the three fundamental cases. We adopted a 2-D time-domain TGEM simulation technique developed by Minami and Toh [2013] to solve equation (1) numerically. Although the method can deal with any tsunami flows, we prescribed a solitary wave with a Gaussian waveform propagating only in the $y$ direction for simplicity, whose waveform and the corresponding seawater velocity can be expressed under the long-wave approximation as

$$
\begin{aligned}
\eta & =A \exp \left(-\frac{(y-c t)^{2}}{2 \chi^{2}}\right), \\
u_{y} & =c \frac{A}{h} \exp \left(-\frac{(y-c t)^{2}}{2 \chi^{2}}\right) .
\end{aligned}
$$

Here $c=\sqrt{g h}$ is the tsunami phase velocity, while $h, A$, and $\chi$ are the ocean depth, the height of the solitary wave, and the horizontal scale length of the tsunami dynamic field. We set $b_{z}$ to zero at the boundaries (the Dirichlet-type condition), approximately $10^{4} \mathrm{~km}$ far from the center grid. The other fields, $b_{y}$ and $e_{x}$, were calculated from the obtained $b_{z}$ using the relations $\nabla \cdot \mathbf{b}=0$ and $\nabla \times \mathbf{e}=-\partial \mathbf{b} / \partial t$. In our numerical simulations, we adopted a homogeneous half-space conductor of $0.01 \mathrm{~S} / \mathrm{m}$ beneath the seafloor and set $F_{z}, \sigma, A$, and $\chi$ to $-35,000 \mathrm{nT}, 4.0 \mathrm{~S} / \mathrm{m}, 1.0 \mathrm{~m}$, and $20 \mathrm{~km}$, respectively. Here we also assumed that $F_{z}$ is both vertically and horizontally constant.

Figure 2 shows the simulation results corresponding to the three fundamental cases, which can be interpreted physically as shown in Figure 3.

\subsection{Diffusion Dominant Case}

The TGEM phenomenon shown in Figure 2 (left) corresponds to Diffusion dominant case $(h / L<0.5)$. When the ocean depth is shallow enough, the diffusion term becomes comparable to the source term, which leads to

$$
0 \approx \nabla \times(\mathbf{v} \times \mathbf{F})+K \nabla^{2} \mathbf{B}
$$

Since the self-induction term is almost negligible, observable magnetic fields are almost the same as those generated by the source current, $\sigma(\mathbf{v} \times \mathbf{F})$. Figure 3 (left) shows Diffusion dominant case as well. The peaks of the downward and upward magnetic components appear almost $90^{\circ}$ ahead of and behind the peak of the sea surface elevation, respectively.

\subsection{Intermediate Case}

Contribution of the self-induction term, $\partial \mathbf{B} / \partial t$, increases with the ocean depth and generates large enough induced electric field, $e_{x}$, through Faraday's Law of induction. TGEM phenomenon where both diffusion and self-induction terms are significant can be referred to Intermediate case $(0.5<h / L<2.0)$. Figures 2 (middle) 

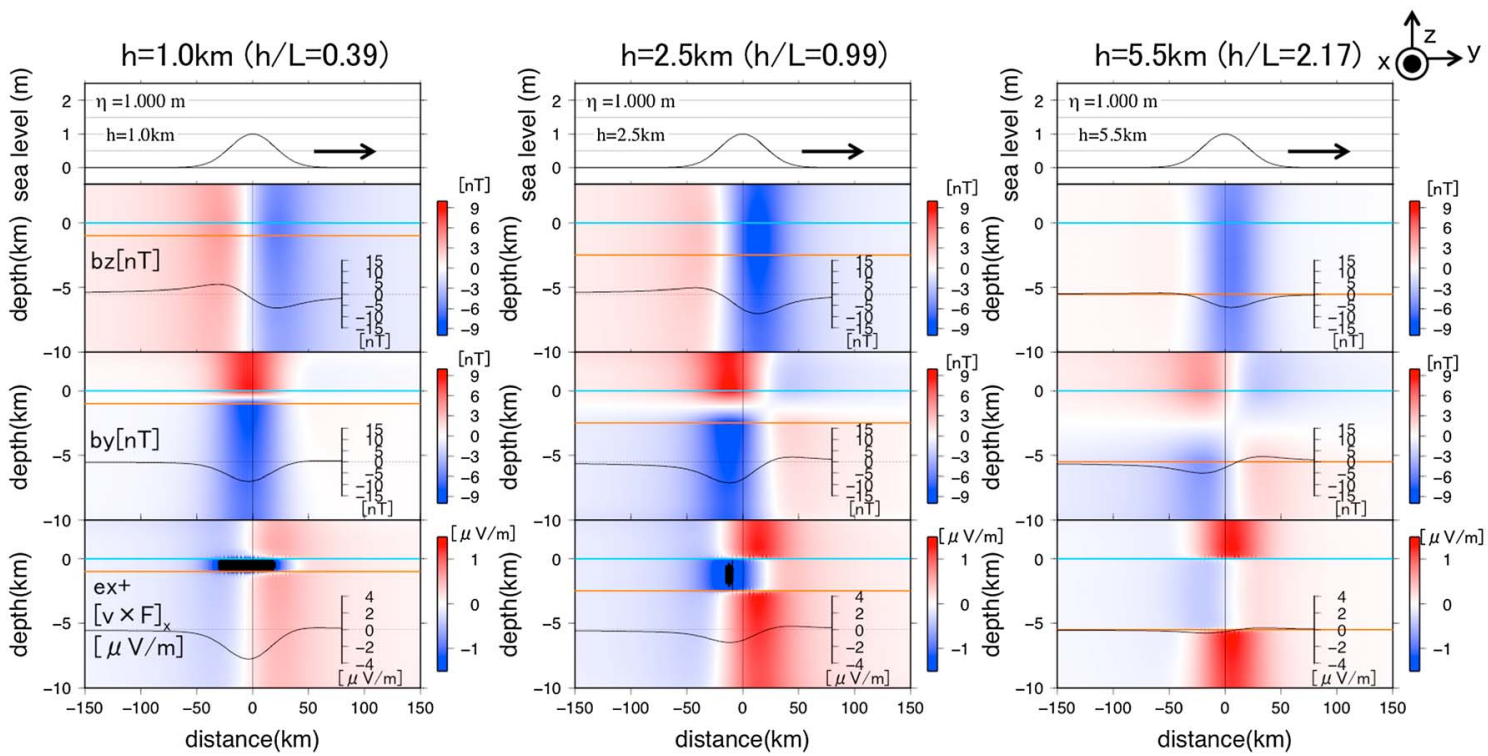

Figure 2. EM fields generated by a rightward propagating solitary tsunami with a Gaussian waveform. Three columns show the results calculated for the three different ocean depths of (left) 1.0, (middle) 2.5 , and (right) $5.5 \mathrm{~km}$ from left to right, respectively. In the second to fourth rows of each column, vertical cross sections of $b_{z \prime}, b_{y}$, and $e_{x}$ $+[\mathbf{v} \times \mathbf{F}]_{X}$ over $z=-10 \mathrm{~km}$ to $2 \mathrm{~km}$ are drawn from top to bottom, where the sea surface $(z=0 \mathrm{~km})$ and seafloor $(z=-h \mathrm{~km})$ are depicted by horizontal light blue and brown lines, respectively. The red and blue colors denote positive and negative values of each EM component, respectively. Profiles of the EM components on the seafloor are also drawn by black solid lines at $z=-5.5 \mathrm{~km}$. The vertical black line corresponds to the center of the Gaussian waveform, where the inducing $\mathbf{v} \times \mathbf{F}$ field is strongest.

and 3 (middle) correspond to this case. The increased self-induction generates $e_{x}$ opposite to $\mathbf{v} \times \mathbf{F}$ and ahead of the solitary wave, which causes not only a delay in phase but also attenuation in amplitude of $b_{z}$ and $b_{y}$ compared to those generated by $\mathbf{v} \times \mathbf{F}$. However, it is noticeable that $e_{x}$ opposite to $\mathbf{v} \times \mathbf{F}$ produces an initial rise in $b_{y}$ in front of the tsunami, which is not present in Diffusion dominant case. This initial rise in $b_{y}$ might be applied to tsunami early warning, because it precedes all the other peaks of TGEM fields and the tsunami arrival itself.
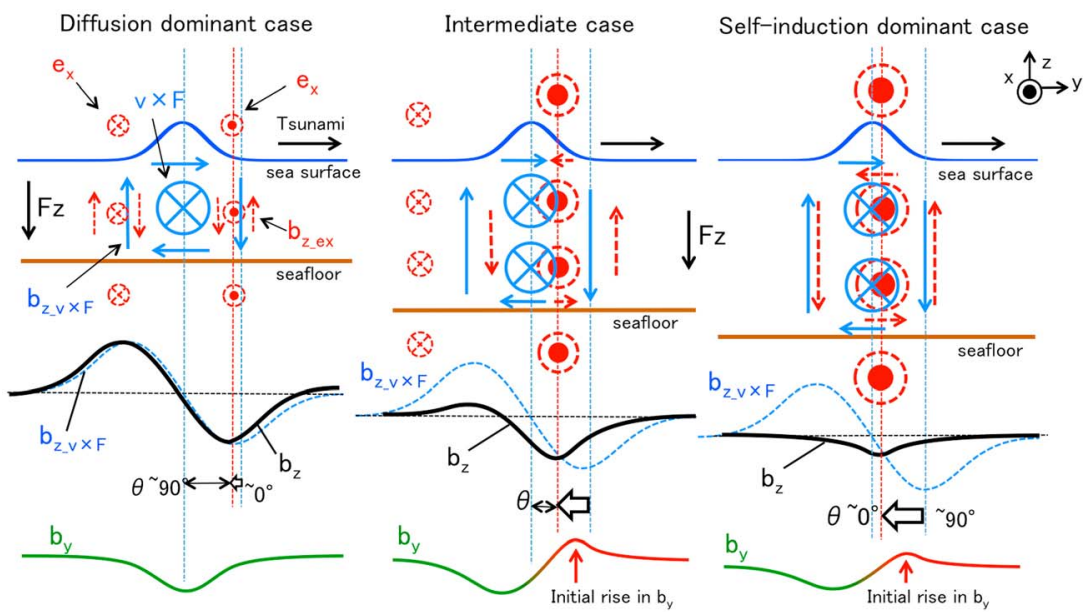

Figure 3. Schematic figures for the three fundamental cases of TGEM phenomena. In the three figures, the blue solid and red dashed arrows in the ocean layer indicate the magnetic fields generated by $\mathbf{v} \times \mathbf{F}$ and that by $e_{x}$, respectively. (left) In the Diffusion dominant case, the self-induction is so weak that the observed magnetic fields $\left(b_{y}, b_{z}\right)$ are virtually equivalent to those generated by $\mathbf{v} \times \mathbf{F}$. (middle) In the Intermediate case, the self-induction effect generates a strong counter electric field $\left(e_{x}\right)$ in front of tsunamis, resulting in phase delay in $b_{z}$ and $b_{y}$. (right) In the Self-induction dominant case, the increased self-induction enables $e_{x}$ to have almost the same magnitude as that of $\mathbf{v} \times \mathbf{F}$, which leads to $b_{z}$ nearly in phase with the sea surface elevation. In both the Intermediate case and Self-induction dominant case, $e_{x}$ opposite to $\mathbf{v} \times \mathbf{F}$ induces an initial rise in $b_{y}$ at the seafloor prior to the tsunami arrival. 


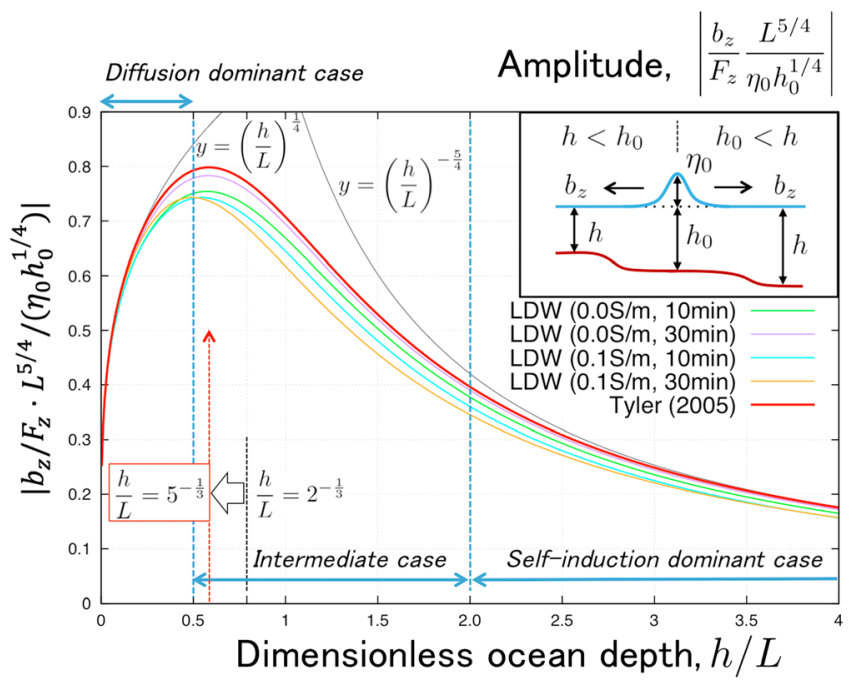

Figure 4. Amplitudes of $b_{z} / F_{z} \cdot L^{5 / 4} /\left(\eta_{0} h_{0}^{1 / 4}\right)$ as a function of the dimensionless ocean depth $(h / L)$. The upper right inset indicates configurations of the two cases, $h<h_{0}$ and $h_{0}<h$. All the LDW lines are calculated for the same parameters as Figure 1 . The vertical dashed black line $\left(h / L=2^{-\frac{1}{3}}\right)$ indicates the peak location of the red line in Figure $1 \mathrm{~b}$. The red solid line here has a peak where $h / L=5^{-\frac{1}{3}} \approx 0.5848$, viz., $c / c_{d}=1 / \sqrt{5}$. The red solid line also asymptotes $y=(h / L)^{1 / 4}$ and $y=(h / L)^{-5 / 4}$ in the Diffusion dominant case and Self-induction dominant case, respectively. Note that all the profiles in Figure 4 approach the origin as $h / L \rightarrow 0$.

\subsection{Self-Induction Dominant Case}

Figure 2 (right) shows the results for the deepest ocean depth of $5.5 \mathrm{~km}$ $(h / L \approx 2.17)$. When the ocean is deep enough $(2.0<h / L)$, we refer to the TGEM phenomenon as Self-induction dominant case, since the diffusion term becomes much smaller than the self-induction term. In this case, the self-induction term alone nearly balances the source term, namely, the frozen-flux approximation holds

$$
\frac{\partial \mathbf{B}}{\partial t} \approx \nabla \times(\mathbf{v} \times \mathbf{B}) .
$$

Figure 3 (right) shows a schematic figure for this case. The strong self-induction induces $e_{x}$ slightly ahead of the tsunami center with as large magnitude as $\mathbf{v} \times \mathbf{F}$, which makes both the total electric field $\left(e_{x}+[\mathbf{v} \times \mathbf{F}]_{x}\right)$ and amplitudes of $b_{z}$ and $b_{y}$ much smaller than those expected without the selfinduction. However, these magnetic components are still observable on the seafloor as reported by Toh et al. [2011]. The $b_{z}$ variation is almost in phase with the sea surface elevation, viz., $\theta \sim 0^{\circ}$, because the phase delay in $b_{z}$ becomes approximately $90^{\circ}$ compared with Diffusion dominant case. The initial rise in $b_{y}$ recognized in Intermediate case still resides in front of the tsunami even in this case. The $b_{y}$ variation prior to the tsunami arrival reported by Minami and Toh [2013] can be interpreted as an initial rise in Self-induction dominant case.

Throughout the three cases, we found that the leading variations in $b_{z}$ are always negative, which stems from the upward positive definition of $b_{z}$ and $F_{z}$ and the negative value of $F_{z}(=-35,000 \mathrm{nT})$. Irrespective of the definition, however, one can generally understand that $b_{z}$ starts to vary in the same direction as the vertical geomagnetic component, $F_{z}$, prior to the arrival of the solitary wave with rightward particle motion. In our calculations, we adopted $F_{z}=-35,000 \mathrm{nT}$ as a typical value at a mean latitude in the Northern Hemisphere. However, the results can be applied to all latitudes (except right at the magnetic equator where the approximations involved in equation (1) break down), with only some changes in the amplitude of $b_{z \prime}$ which is proportional to $\left|F_{z}\right|$ as anticipated by equations (2) and (3).

\section{Effects of Ocean Depth Change on Tsunami Propagation}

In the above investigation of the dependence of TGEM fields on the ocean depth, the tsunami height was kept constant. Here we revisit the amplitude problem of TGEM fields for variable ocean depth, applying the concept of the conservation of hydrodynamic energy flux of each tsunami. It is known, to first approximation, that the energy flux of tsunamis is preserved during propagation at any ocean depths. Assuming a long plane wave with an amplitude of $|\eta|$ over an ocean depth of $h$, the energy flux of tsunamis is expressed as a product of the phase velocity and its wavelength-averaged dynamic energy, $\sqrt{g h} \times(1 / 2) \rho g|\eta|^{2}$, where $\rho$ is the seawater density. The conservation of this energy flux leads to the following relationship between the tsunami height and the ocean depth:

$$
|\eta|=\left(h_{0} / h\right)^{\frac{1}{4}}\left|\eta_{0}\right|
$$

Here $h_{0}$ and $\left|\eta_{0}\right|$ are the ocean depth and the tsunami height at a reference point, respectively. Equation (11) is valid independent of tsunami propagation process between the two constant ocean depths, $h_{0}$ and $h$, 
unless the tsunami loses its dynamic energy due to, for instance, reflection. Substituting equation (11) into equations (2) and (3) yields new functions of $\left|b_{z}\right|$ in terms of the ocean depth. As for equation (6), the source current term, $\int_{-h}^{0} i_{Q}=0 \mathrm{~d} z \propto h^{1 / 2}$, is replaced by

$$
\int_{-h}^{0} i_{Q=0} \mathrm{~d} z=\int_{-h}^{0}\left[\sigma F_{z} c \frac{\left|\eta_{0}\right|}{h}\left(\frac{h_{0}}{h}\right)^{1 / 4}\right] \mathrm{d} z \propto h^{\frac{1}{4}} .
$$

Figure 4 shows the dependence of dimensionless amplitude, $\left|b_{z} / F_{z} \cdot L^{5 / 4} /\left(\eta_{0} h_{0}^{1 / 4}\right)\right|$, on the dimensionless ocean depth, $h / L$, calculated by equations (2) and (3) with equation (11). For comparison, the peak location of the red solid line in Figure $1 \mathrm{~b}\left(h / L=2^{-\frac{1}{3}}\right)$ is depicted as a black dashed line in Figure 4. We can see that the peak of the red solid line in Figure 4 is located at a shallower depth than that in Figure $1 b$, which is due to tsunami height enhancement in shallow oceans. The peak of the red line in Figure 4 is located at $h / L=5^{-\frac{1}{3}} \approx 0.5848$, viz., $Q=c / c_{d}=1 / \sqrt{5}$, which corresponds to $h \approx 1.5 \mathrm{~km}$ when we assume $L=2.53 \mathrm{~km}$. In the Diffusion dominant case, the red line asymptotes at $y=(h / L)^{1 / 4}$ because $1 /(Q+i) \sim-i$ and $\int_{-h}^{0} i_{Q=0} \mathrm{~d} z \propto h^{1 / 4}$, while it asymptotes at $y=(h / L)^{-5 / 4}$ in the Self-induction dominant case, because $1 /(Q+i) \sim Q^{-1} \propto h^{-3 / 2}$ and $\int_{-h}^{0} i_{Q=0} \mathrm{~d} z \propto h^{1 / 4}$ in deep oceans.

\section{Discussions and Summary}

In this paper, we provide a classification of TGEM phenomena into three cases based on the ocean depth. From shallow to deep ocean depths, they are the Diffusion dominant case, Intermediate case, and Selfinduction dominant case. Using this classification, it was shown that the self-induction is stronger with increasing ocean depths, the phase lead of $b_{z}$ to $\eta$ is smaller in deeper oceans, and the amplitudes of the TGEM fields are largest in the Intermediate case (see Figure 1). Furthermore, our numerical simulations showed that the $b_{z}$ variation is observed prior to tsunami arrivals in the Diffusion dominant case, while an initial rise in $b_{y}$ precedes all the other EM signals in the Intermediate case and Self-induction dominant case (see Figures 2 and 3).

In section 4, we investigated the dynamically consistent effects of ocean depth change on TGEM fields. As a result, it was found that Figure 4 is a more accurate description of real phenomena than Figure $1 \mathrm{~b}$. It is noteworthy that 2-D TGEM fields are likely to have largest amplitudes not at coastlines but offshore, or around $h / L=5^{-\frac{1}{3}}$, viz., for an ocean of $h \approx 1.5 \mathrm{~km}$ and $\sigma=4.0 \mathrm{~S} / \mathrm{m}$, while tsunami wave heights usually become highest at coastlines. At an ocean depth of approximately $1.5 \mathrm{~km}$, where the amplitudes of TGEM fields are largest in the course of tsunami propagation, the peak of $b_{z}$ precedes that of $\eta$ by $\sim 0.18 T$ (T: tsunami period), since $h / L=5^{-\frac{1}{3}}$ corresponds to $\theta \approx 66^{\circ}$ (see Figure 1a). On the other hand, without the dynamical effect of ocean depth changes, we have maximum amplitudes around $h / L \sim 2^{-\frac{1}{3}}$, viz., $h \sim 2.0 \mathrm{~km}$ (see Figure $1 \mathrm{~b}$ ), where $\theta \approx 55^{\circ}$ results in $b_{z}$ preceding $\eta$ by $\sim 0.15 T$ (see Figure 1a). When $T=30 \mathrm{~min}$, the time lead of $b_{z}$ relative to $\eta$ at $h=1.5 \mathrm{~km}, \sim 5.4 \mathrm{~min}$, is only $\sim 1 \mathrm{~min}$ earlier than that at $h=2.0 \mathrm{~km}, \sim 4.5 \mathrm{~min}$. We, therefore, can conclude that not only around $1.5 \mathrm{~km}$ but also up to $2.0 \mathrm{~km}$ deep oceans are good candidates for seafloor EM observations aimed at tsunami early warning, because $\sim 5 \mathrm{~min}$ time lead of $b_{z}$ to $\eta$ allows us early tsunami detection prior to tsunami arrivals and amplitudes of tsunami magnetic signals are close to peak values. Although all the analyses in this paper are limited to 2-D configuration, they may be valid in the vicinity of coastlines where wave fronts tend to face coastlines due to refraction, or for large-scale tsunamis that can be approximated by plane waves.

Acknowledgments

This work is supported by Grants in Aid for Scientific Research of MEXT, Japan (13J01475 and 26282101). T.M. expresses his sincere thanks to Weijia Kuang and other colleagues in the NASA Goddard Space Flight Center (GSFC) for their warm support to T.M.'s study in GSFC.

The Editor thanks Angelo De Santis and an anonymous reviewer for their assistance in evaluating this paper.

\section{References}

Ichihara, H., Y. Hamano, K. Baba, and T. Kasaya (2013), Tsunami source of the 2011 Tohoku earthquake detected by an ocean-bottom magnetometer, Earth Planet. Sci. Lett., 382, 117-124, doi:10.1016/j.epsl.2013.09.015.

Larsen, J. C. (1971), The electromagnetic field of long and intermediate water waves, J. Mar. Res., 29, 28-45.

Minami, T., and H. Toh (2013), Two-dimensional simulations of the tsunami dynamo effect using the finite element method, Geophys. Res. Lett., 40, 4560-4564, doi:10.1002/grl.50823.

Sugioka, H., Y. Hamano, K. Baba, T. Kasaya, N. Tada, and D. Suetsugu (2014), Tsunami: Ocean dynamo generator, Sci. Rep., 4, 3596, doi:10.1038/srep03596. 
Toh, H., K. Satake, Y. Hamano, Y. Fujii, and T. Goto (2011), Tsunami signals from the 2006 and 2007 Kuril earthquakes detected at a seafloor geomagnetic observatory, J. Geophys. Res., 116, B02104, doi:10.1029/2010JB007873.

Tyler, R. H. (2005), A simple formula for estimating the magnetic fields generated by tsunami flow, Geophys. Res. Lett., 32, L0960, doi:10.1029/2005GL022429.

Zhang, L., K. Baba, P. Liang, H. Shimizu, and H. Utada (2014a), The 2011 Tohoku Tsunami observed by an array of ocean bottom electromagnetometers, Geophys. Res. Lett., 41, doi:10.1002/2014GL060850.

Zhang, L., H. Utada, H. Shimizu, K. Baba, and T. Maeda (2014b), Three-dimensional simulation of the electromagnetic fields induced by the 2011 Tohoku tsunami, J. Geophys. Res. Solid Earth, 119, 150-168, doi:10.1002/2013JB010264. 